\title{
Las mujeres latinoamericanas: generación de datos y metodología para investigaciones futuras*.
}

\section{Carmen Ramos Escandón}

In relación con la investigación sobre mujeres, hace más de diez años se puntualizó: "lo que destacó en las discusiones habidas en la reunión de la ciudad de México, fue la importancia de apartarse de esquemas de análisis de tipo genérico, para buscar soluciones específicas que puedan aplicarse a una sociedad y grupo social particular. Al mismo tiempo se hizo obvio que muchos de los temas están estrechamente interrelacionados y que deben contemplarse desde una perspectiva de tiempo amplia, así como compararse en diferentes sociedades".'

Hagamos pues una primera observación sobre la importancia y validez de este enunciado en la actualidad. De hecho, en los últimos diez años se ha acentuado la necesidad de analizar la situación de las mujeres dentro de una perspectiva de tiempo amplia, es decir, desde un punto de vista histórico que nos permita reconocer, por medio de la variedad de experiencias femeninas, las continuidades y los cambios en sus funciones y actividades en el curso de la historia.

Es necesario conocer las causas de los cambios más importantes en la vida de las mujerés; cómo incorporan las mujeres esos cambios en su vida, si su actividad cotidiana se modifica y cómo, en qué medida, con qué ritmo, por qué. Sin embargo, para partir de una perspectiva válida, es necesario ser capaces de identificar la relación entre cambio y continuidad en la posición de las mujeres, en sus vidas, en sus funciones sociales, en los estereotipos femeninos que definen el "Ser Mujer".

El tiempo, esa materia prima de la historia, es un elemento im-

La versión inglesa de este artículo se presentó en la reunión de la uNESCo, Paris, noviembre 13,16, 1984: "Meeting of experts on Theoretical frameworks and methodological approaches to studies on the role of women in history as actors in economic, social, political and ideological processes." SHS 84 Conf Pop/1.03. La traducción es de la autora.

' Hanna Papaneck, "The work of women. Postcript from Mexico City" en SIGNS, núm. 1, vol. 1, otoño 1975, p. 225. Papaneck discutió la importancia de las diferencias sociales entre las mujeres y la necesidad de entenderlas en términos de "los factores estructurales, relacionando las variaciones políticas y económicas en un contexto cultural".

- En general, como una gran simplificación, puede decirse que el principal papel histórico de las mujeres ha sido el de la reproducción de la especie, y sin embargo, aun una función de tal continuidad biológica ha sido objeto de cambios a través de la historia. El historiador inglés Edward Shorter ha publicado recientemente un libro en el que se ocupa precisamente de las prácticas respecto al parto en Europa, desde la edad media hasta el siglo XIX. Véase: Shorter, Edward $A$ history of $W o-$ man's bodies. Londres, Pengüin, 1982. 
portante que debe considerarse al analizar a las mujeres, pues precisamente por el hecho de que sus papeles y actividades hayan sido ignorados por tanto tiempo, puede llevar a la simplificación de que la historia de las mujeres es repetitiva, que "nada cambia en su vida". Precisamente por eso necesitamos tomar en consideración grandes espacios de tiempo para superar un enfoque de meros acontecimientos en lo que se refiere a la historia de las mujeres. Al analizar el periodo en relación con una perspectiva secular, estaremos en condición de localizar los momentos de cambio, a la vez que las continuidades y permanencias. ${ }^{3}$ Esto añade complejidad al problema pero enriquece nuestra perspectiva y la hace más exacta.

Otro elemento que debe tenerse en cuenta en relación con la historia de las mujeres es su especificidad; el hecho de que frecuentemente la experiencia histórica de la humanidad se ha enunciado hasta ahora sólo en términos de historia de los hombres. Desde luego que coexisten hombres y mujeres en la historia, pero hasta hoy, únicamente se ha considerado la actividad masculina como materia de estudio histórico, por ejemplo en la política, en la vida militar o en la actividad económica asalariada. $\mathrm{Ni}$ qué decir que este tipo de enfoque nos dé una visión fragmentada de la vida humana, pues simplemente deja de lado la actividad de la mitad de la población. ${ }^{4}$ Sin embargo, uno debe preguntarse si los temas y problemas que son relevantes para los hombres son igualmente relevantes para las mujeres. En otras palabras, tenemos que evaluar la especificidad de la experiencia histórica femenina. Esto puede, de hecho, llevarnos a reformular nuestra concepción de la historia, para darnos cuenta que hechos tales como los esquemas reproductivos, las prácticas de crianza de infantes, las formas de organización de la unidad doméstica, los procesos de preparación de alimentos y otras "actividades femeninas" son materias legitimas de análisis histórico y ciertamente, un importante elemento para entender las sociedades humanas y las formas de organización social de los grupos humanos en el pasado.

El hecho de que las mujeres no pertenezcan a una sola clase, sino que son miembros de diferentes grupos sociales o clases que coexisten en un tiempo y lugar específicos, añade complejidad al problema; sin embargo, es necesario tratar de entender cómo se construye históricamente la identidad femenina y sus variaciones a través del tiempo, de las clases, de las religiones, etc.

Este tipo de análisis es aún embrionario en la historiografia latinoamericana. Lo que existe es un enfoque tradicional en el cual

En relación al problema de la larga duración véase: Fernand Braudel, La historia y las ciencias sociales, Madrid, Alianza Editorial, 1974. Especialmente el capitulo III "La larga duración", p. 60-106. En relación con las mujeres es particularmente importante senalar que puesto que han sido consideradas meramente como instrumentos reproductivos, y su situación de sumisión se explica frecuentemente en relación con los papeles que se les asignan en la familia, es importante analizar las diferencias en esas funciones a través del tiempo, en especial la relación entre el tiempo individual, personal e industrial. Véase: Tamara Hareeven, Family Time and Industrial time. Nueva York, Cambridge University Press, 1982.

Mary Nash. "Nuevas dimensiones en la historia de la mujer" en Presencia y Protagonismo. Aspectos de la historia de la mujer. Barcelona, Ediciones del Serbal, 1984. p. 9-50. También el volumen dedicado a la historia de la mujer de la revista The American Historical Review; num. 89, vol. 3, junio de 1984. 
se resaltan las virtudes de las mujeres notables: reinas, heroínas que se enfocan y se destacan históricamente en razón de su carácter excepcional. Sin embargo, es muy frecuente juzgar que lo que las hace notables es el hecho de que obedecen a conductas no femeninas, que actúan contra las normas establecidas, prescritas como propias de las mujeres en su época, y se les considera notables porque se les mide en razón de parámetros de conducta masculinos, no femeninos.

Otro importante enfoque que ha prevalecido en el estudio de las mujeres ha sido el del efecto de espejo; es decir que las mujeres se vuelven notables por su asociación con un hombre, en una empresa en común o, más frecuentemente, en una relación amorosa. En América Latina, Malintzin, la amante del conquistador de México, Hernán Cortés, es un caso característico de este punto de vista. En otro momento, varios siglos después, Manuela Sáenz es otra mujer que alcanzó celebridad por ser la amante de un héroe de la independencia latinoamericana: Simón Bolivar. La bibliografia es abundante y a menudo descansa en la descripción de las particularidades del episodio amoroso o del escándalo que produjo en la época. ${ }^{5}$

Son estas perspectivas las que, una vez más, limitan la importancia de la mujer, supeditándola a alguien más, alguien perteneciente al sexo masculino, desde luego, negándole su ser como.individuo, su derecho a ser parte de la historia en sus propios términos.

Sin embargo, el tiempo y el carácter específico no son los únicos elementos que deben contar en la historia de las mujeres. Necesitamos saber más respecto a los problemas relevantes y significativos para analizar la historia de mujeres, problemas que adquirirán mayor peso si se les añaden las variaciones étnicas, sociales y regionales como elementos importantes que den razón de las diferencias en los esquemas de sus vidas. ${ }^{6}$ Tenemos que medir dichos elementos uno frente al otro para poder identificar tendencias y variantes.

Es precisamente el caso latinoamericano el que mejor se presta a un acercamiento de esa naturaleza. A través de "la larga duración", los diferentes aspectos de la vida de las mujeres de la región pueden analizarse, tanto por sus similitudes como por las variantes regionales, sociales y étnicas. ${ }^{7}$

${ }^{5}$ Para una bibliografía sobre ambas mujeres, véase: Meri Knaster, Women in Spanish America, Boston, A. K. Hall, 1977, p. 456-459 y 493-500.

- Véase Michele Perrot, editora, ¿Une histoire de femmes, est-elle possible?, París, Rivages, 1984.

"Asunción Lavrin, "Recent studies on women in Latin America" en Latin American Research Review; vol. XIX, núm. 1, 1984, p. 181-190. June Hanher, editora, Women in Latin American History, Los Angeles, UCLA, Latin American Center Publications, 1980, p. 1-17. Susan Soeiro, "Recent work on Latin American women: a review essay", Jottrnal of Interamerican Studies and World Affairs, vol. 17, nov. 1975, p. 497-516. Meri Knaster, "Women in Latin America: The state of research, 1975 en Latin American Research Review," vol. XI, primavera 1976, p. 3-74. Otilia De Tejeira, The women in Latin America: past, present, future, Washington, Organización de Estados Americanos. Comisión Interamericana sobre mujeres, 1928-1973, 1974. Ann Pescatello, "The female in Iberoamerica, an essay research on bibliography and research directions" en Latin American Research Review; vol. 7 , verano 1972 , p. 121-141. 
El beneficio de dicha perspectiva es el de permitirnos evaluar adecuadamente la importancia de la participación femenina en los procesos sociales, políticos y económicos de la experiencia latinoamericana.

En otras áreas, sobre todo en los Estados Unidos y en Europa, y particularmente en Inglaterra, una generación de historiadores jóvenes se ha ocupado de la historia de las mujeres considerándola un campo de investigación importante. En América Latina la investigación en ese sentido es aún escasa, fragmentaria y frecuentemente difícil de encontrar. Esta razón puede tener dos causas principales: el que los escasos recursos hayan privilegiado otras áreas del estudio de la mujer, puesto que la historia femenina hasta el momento no es una disciplina que tenga prioridad en la política de investigación. Otra razón importante para esta escasez de estudios puede ser el hecho de que se trata de seguir al pie de la letra las pautas de investigación europea o norteamericana que están destinadas al fracaso. Las fuentes son diferentes, los problemas son diversos y las perspectivas e interpretaciones tienen que reflejar estas diferencias. Es necesario, para los que se interesan en la mujer de América Latina y en su historia, descubrir con una mirada nueva y fresca, los problemas y los temas de investigación. El primer paso en este nuevo camino sería el del descubrimiento. ${ }^{8}$ Como muchos otros actores invisibles en la historia de América Latina, las mujeres deben cruzar el largo camino de la invisibilidad para alcanzar el nítido perfil de su presencia, de tal manera, que se pueda reconocer a la mujer de la América Latina contemporánea en el contexto de su historia particular, que le permita reconocerse y aceptarse con sus propias particularidades, reconociendo y asimilando una herencia que hasta ahora no forma parte de su conciencia histórica.

Debemos saber más de la historia de la mujer latinoamericana

\footnotetext{
* Por ejemplo, una de las fuentes más importantes para la historia de las mujeres ha sido el hallazgo de diarios personales y cartas de mujeres. Algunos ejemplos al respecto son Harriet H. Robinson, Loom and Spindle, Hawai, Press Pacífica, 1976, o recientemente el caso de las cartas del siglo diecinueve publicadas como: Anónimo, Marthe, París, Seuil, 1982. Este tipo de fuentes han sido bastante escasas en América Latina, puesto que en un continente con bajos indices de alfabetización, los diarios de las mujeres no son tan abundantes, dado que hasta fines del siglo pasado eran proporcionalmente pocas las mujeres que sabian leer y más aún escribir. En América Latina, es muy posible que la tradición de tipo oral resulte más importante. Algunos ejemplos sobresalientes de diarios femeninos son: Tristán y Moscozo, Flore Celestine, Térese Henriette, Peregrinaciones de una paria, Santiago de Chile, Ediciones Ercilla, 1941. Originalmente escrito en francés con el título Peregrinations d'une paria entre 1833 y 1834 , y publicado en 1838 en París. Véase también Concèpción Lombardo de Miramón, Memorias, México, Editorial Porrúa, 1980; Villaseca, editor, Cartas de Mariquita Sánchez, Buenos Aires, Ediciones Peusser, 1952. Otra fuente para la historia de las mujeres son los testimonios de viajeras y viajeros que recorrieron América Latina durante el siglo pasado. Algunos de los más famosos, son: Frances E.. Calderón de la Barca, Life in Mexico during a residence of two years in the country, con prefacio de $W$. $H$. Prescott, London, Chapman Hall, 1843, que cuenta con varias ediciones en español. Maria Calcott Graham, Diario de su viaje de residencia en Chile y de su viaje a Brasil, Madrid, Editorial América, s. f. Sobre la importancia de la historia oral para el estudio de la historia femenina véase: Sylvie Van de Casteele y Danièle Voldan, "Les sources orales pour l'histoire des femmes" en Perrot, Michèle editor. ¿Une historie des femmes, est-elle possible?, Paris, Rivages, 1984, p. 57-70.
} 
para entendernos mejor, como mujeres y como latinoamericanas. Dado que América Latina es un continente de amplia variedad humana y geográfica, debe reconocerse el hecho de que es producto de una vasta herencia cultural, de que sus pueblos viven simultáneamente varios estadios de desarrollo histórico, por lo que podríamos empezar por elaborar un esquema de periodización que nos permita tomar en cuenta esas diferencias, especialmente entre varios grupos de mujeres.

En ese sentido, un primer tipo de periodización podría establecerse en relación al modo de producción dominante y cómo han afectado esos cambios a la mujer. Hace cuarenta años se inició el debate sobre la naturaleza de la economia latinoamericana en términos de feudalismo y capitalismo ${ }^{9}$ y las diferentes respuestas a esta cuestión han subrayado el carácter de la fuerza de trabajo o de la circulación como el elemento determinante para definir la . naturaleza de la economía latinoamericana. ${ }^{10}$ Sin embargo, muy pocas veces se ha subrayado el hecho de que mucho del trabajo no asalariado en la economia campesina ha sido, y es, trabajo femenino."

Las formäs complejas en que el trabajo femenino y masculino se mezclan en la economía campesina latinoamericana y la relación entre propietarios privados y comunidades, aún no han sido suficientemente analizadas en lo que se refiere al trabajo de las mujeres. ¿Mejoraron las mujeres su situación cuando las tierras comunales se hicieron privadas o se les privó de su papel tradicional en la comunidad? ¿Cómo han cambiado los esquemas de reproducción con los rápidos cambios económicos de la región? Muchas preguntas que se han analizado en el caso de la América Latina contemporánea, tienen hondas raíces en el pasado y solamente si recordamos que la situación actual de la mujer latinoamericana es el producto de una experiencia acumulada, podremos enfrentar y evaluar nuestro pasado y, en la medida de lo posible, elegir nuestro futuro.

Aunque han sido muchas las mujeres que en la historia latinoamericana han tenido un papel importante en diferentes aspectos del proceso productivo, su contribución económica no ha sido reconocida hasta hace muy poco. La mayoria de las veces, se considera a la mujer económicamente activa sólo si participa en actividades asalariadas, por lo cual contamos aún con una imagen sumamente fragmentada e incompleta de la participación econó-

\footnotetext{
"Véase por ejemplo. Sergio Bagú, Feudalismo y Capitalismo en la historia de America Larina. Buenos Aires, 1946.

"1 Para una sintesis de la polémica sobre la naturaleza de la economía latinoamericana véase: Marco Ingrosso, Modelos socioeconómicos de interpretación de la realidad latinoamericana. de Mariátegui a Gunder Frank, Barcelona, Anagrama. 1973. Cuadernos Anagrama. núm. 62: también Ernesto Laclau. Critica sobre el orige'n y naturaleza social de Latinoamérica, Buenos Aires, Antigua Casa. Editorial Cuervo. 1977. Para uria discusión histórica de los modos de producción en América Latina véase: Sempat Assadpurian, y otros, Modos de Prodicción en América Latina. México. Siglo XXI. 1977. Cuadérnos de pasado y presente, núm. 40.

11 Para una discusión reciente sobre el trabajo femenino en América Latina. véase: Eleanor Leacock, y Helen Safa, Women's work development and the division of lahor by' gender. Massachussetts, Bergin and Garvey, 1985. Para el estudio de las funciones femeninas y sus cambios véase: June Nash, Helen Safa and contributors, W'omen and Change in Latin America, Massachussetts. Bergin and Garvey, 1985.
} 
mica de las mujeres en la región, particularmente en épocas pasadas. $^{12}$

Es necesaria una mayor y más profunda investigación en la cual las mujeres sean analizadas en razón de los diferentes aspectos de su actividad económica, en el hogar y fuera de él; los tipos de trabajos que llevan a cabo, los grupos sociales a los que pertenecen y las influencias ideológicas a las que están sujetas. Todos estos aspectos de la vida de las mujeres tienen una correlación entre sí, y es necesario conocer las características de esa correlación. Al mismo tiempo, debemos establecer también las diferencias entre las actividades que socialmente se han adscrito a las mujeres y la percepción femenina de esas actividades y estereotipos, y cómo se pliegan a ellos las mujeres o si los rechazan.

Es necesario superar la noción que se tiene, en los países sajones, sobre todo, de la mujer latinoamericana en general, para tratar de identificar las diferencias regionales. La herencia histórica de las mujeres de países con una abundante población indígena como Bolivia, Colombia, Ecuador, Guatemala, Perú y México, debe ser analizada tomando en consideración el hecho de que las culturas indígenas y su organización social han tenido, a lo largo de la historia, un papel muy importante en la vida cotidiana de las mujeres de estas áreas. ${ }^{13}$ Esta herencia indígena va mucho más allá de aspectos como la vestimenta, la comida o las prácticas religiosas, y tiene una incidencia importante en aspectos tales como la organización del trabajo, las prácticas reproductivas y las actitudes politicas, entre otras.'

En otro tipo de países latinoamericanos como Argentina, Uruguay y Chile, las mujeres de esas regiones deberán ser analizadas frente al hecho de que estos países son producto de una emigración masiva, mayoritariamente de la Europa mediterránea. Allí, la herencia cultural está más relacionada con las tradiciones euro-

12 El caso andino parece ser una excepción. Alli existe un grupo de historiadores que han reconstruido cuidadosamente la organización económica de las comunidades indígenas y la relación entre las comunidades y la sociedad blanca; en este tipo de investigaciones, el trabajo de las mujeres en las comunidades ha sido analizado en sus raíces históricas. Véase: Florencia Mallon, "Gender and class in the transition to capitalism: Housenhold and mode of production in Central Perú 18601950". Trabajo presentado en la reunión sobre Género y desigualdad social de clase en América Latina, México, Social Science Research Council, octubre de 1983. La discusión sobre si las categorías de análisis tradicional son o no válidas para las formaciones precapitalistas en América Latina en relación con el papel de las mujeres en la producción ha sido desarrollado por Carmen Diana Deere en "Changing social relations of production and peruvian peasant woman's work" en Women in Latin America, Riverside, Latin America Perspectives, 1979, p. 26-46. El impacto de la modernización en las funciones asignadas a las mujeres está revisado en June Nash, y Helen Safa, Sex and class in Latin America, segunda edición. Amherst; Mas, J. F. Barguin Publishers, 1980. Véase especialmente el articulo de June Nash "A critique of social roles in Latin America".

is Véase por ejemplo: Lois Paul, "The mastery work and the mystery of sex in a Guatemala Village" en Rosaldo, Michelle y Louis Lampere, Women, culture and society, Stanford, University Press, 1974, p. 281-299.

14 Por ejemplo, un importante fenómeno, el marianismo está presentado como la respuesta de las mujeres latinoamericanas al machismo en Evelyn Stevens, "Marianismo, the other face of machismo in Latin America" en Female and Male in Latin America. Ann Pescatello editor, Pittsburg, University of Pittsburg Press, 1973, p. 90-101. Véase también: Ann Pescatello, editor Power and Pawn: the female in Iberia families, societies and cultures, Wesport, Greenwoodpress, 1976. 
peas y algunas similitudes prodrian encontrarse en aspectos como las normas culturales, las funciones internas de la familia, los tipos de trabajo femenino y la organización política de mujeres.

Brasil podría considerarse un caso en sí mismo, puesto que allí la importancia de la herencia ibérica (Portugal) está disminuida en cierto sentido por la herencia negra, muy importante, sobre todo en la zona del noroeste, región empobrecida agrícolamente a partir de fines del siglo XVIII por la decadencia de la economía de plantación de la caña de azúcar. En esas áreas, la diferencia entre negras, mulatas y blancas es de notarse, como lo es la herencia de la economía de plantación con fuerza de trabajo esclava, para explicar la situación de las mujeres de esa región y sus patrones culturales.

En relación con estas diferencias que se dan sobre todo por el color, Gilberto Freyre desarrolló su interpretación de la historia brasileña y de las relaciones sociales como el producto de las existentes entre propietarios y esclavos. Freyre establecía, ya en los años treinta, que la mujer indígena, especialmente la mujer negra y esclava, fue la que estableció, en el Brasil colonial, las prácticas. de higiene y eróticas que prevalecen aún en la actualidad. ${ }^{15}$

Los estados sureños de Santa Catarina, San Paulo, Paraná y Río Grande del Sur fueron influidos por la emigración, durante el siglo $\mathrm{XIX}$, de campesinos alemanes, suizos, polacos, y aun rusos que se dedicaron al cultivo del café, como en San Paulo y Paraná, o a la crianza de ganado, como en Río Grande del Sur y Santa Catarina. Aquí la cuestión seria analizar hasta qué punto las prácticas europeas de trabajo y vida familiar sobrevivieron entre las mujeres recién emigradas y cómo se modificaron en el nuevo ambiente. Cuál era la relación entre las mujeres recién emigradas y los pobladores ya establecidos y cómo se modificaron los hábitos matrimoniales entre esos grupos diversos. Si hubo emigración de mujeres solas, si fue ese el caso, con qué características y cómo se establecieron las mujeres en estas regiones. Qué similitudes existen entre estas regiones y otras áreas del continente que también han sido zonas de gran inmigración, como la Argentina o el oeste norteamericano. ¿Cómo podemos explicar esas semejanzas y diferencias?

El caso del Caribe es aún más complejo. Allí, la herencia negra es tan fuerte como en Brasil, y la región se orienta en general hacia los cultivos de exportación, con un sistema de producción esclavista. La complejidad se incrementa porque los poderes imperialistas españoles, franceses, holandeses, ingleses y norteamericanos han establecido, con el transcurso del tiempo, diferentes formas económicas y culturales de colonización que afectan a las mujeres de manera diversa. La variedad cultural provee muchos estu-

\footnotetext{
${ }^{15}$ Gilberto Freyre, The masters and the slaves, Nueva York. Alfred A. Knopf, 1956. El titulo original: Casa Grande e Senzala, fue publicado originalmente por José Olympo, Rio de Janeiro, 1933. Para una investigación moderna que corrige la perspectiva de Freyre véase: Charles Boxer, Women in overseas Iberian expansion, Nueva York, Oxford University Press, 1968, p. 345-348. Sobre la esclavitud en hispanoamérica véase: Rolando Mellafe, La esclavitud hispanoamericana, Editorial Universitaria de Buenos Aires, 1972.
} 
dios de casos en los cuales, dentro de un esquema general de carácter similar, las diferencias culturales son enormes. ${ }^{16}$

Sin embargo, para el caso del Caribe, como para el resto de América Latina, debemos darnos cuenta de que las variantes regionales no necesariamente obedecen a diferencias que puedan delimitar una frontera; por el contrario, las fronteras politicas son poco relevantes y las semejanzas y diferencias tienen mucho más que ver con los factores económicos, como en el sur de Brasil, cuyas ligas con Argentina y Uruguay y con otras regiones ganaderas, como los llanos venezolanos, son sumamente claras. Este tipo de región puede compararse en términos económicos y sociales más que nacionales.

Otro caso similar es el de la economía de plantación, que se localiza tanto en el Caribe como en el continente. En el primer caso se trata de Cuba, Puerto Rico, Jamaica, Santo Domingo y Haití: en el continente, en regiones como el noreste brasileño, el sureste mexicano o la costa colombiana. Sin embargo, la perspectiva regional no es la única válida en el análisis de la mujer dentro de su propio contexto.

En una sociedad altamente dividida en grupos sociales, como lo es la sociedad latinoamericana, el factor de la clase a la que pueda pertenecer la mujer tiene enorme importancia. Ignorar las diferencias sociales al analizarla puede llevarnos a caer en estereotipos y nos impediria entender que estas diferencias están profundamente enraizadas en la historia.

Hasta hace muy poco, una falla en los estudios de la mujer en América Latina es la de analizarla en el muy limitado marco de su integración o no integración a un sector moderno de la economía. Con esta perpectiva, la historia de las mujeres en América Latina se ha hecho sumamente estática, especialmente porque parece prevalecer la idea de que las mujeres no son importantes; de hecho ni siquiera se toma en cuenta su presencia, si no están integradas al trabajo asalariado. Sin embargo, las mujeres han tenido un pàpel en la historia latinoamericana y precisamente es necesario identificar y evaluar las modalidades de sus aportes y señalar los aspectos en que éstos han sido de mayor trascendencia.

Puede decirse que además de la producción agrícola, otras áreas en que las mujeres-han desempeñado un papel principal por su integración al trabajo productivo, ha sido en la producción textilera, especialmente en los talleres artesanales, mucho más que en la producción industrial, así como en la manufactura del tabaco, que por mucho tiempo fue una actividad esencialmente femenina, y especialmente en los diversos aspectos de la costura, ya que el bordado. la elaboración de encajes o el trabajo de modista, ha sido la actividad por excelencia de las mujeres de la región. Conocido como el trabajo de la aguja en Cuba o Puerto Rico, el bordado ha

\footnotetext{
to Véase: Frances Henry. "The status of women in Caribbean societies: an overview of their social, economic and sexual roles". Social and Economic Studies, vol. 24 . junio de 1975 , p. 165 . El hecho de que esta área haya sido el lugar donde la colonización europea se originó y de que en la región se puedan rastrear las influencias francesas, holandesas, inglesas, españolas y norteamericanas, para no mencionar a los muchos grupos de origen africano llevados a esa región, nos da idea de la complejidad cultural de la misma, por lo cual esta área merece cuidadosos estudios. con una perspectiva comparativa que nos permita analizar las diferencias en las funciones de las mujeres.
} 
sido tradicional en el área. En Brasil, la elaboración de encajes es de raigambre antigua en la zona bahiana y, en México, las costureras a destajo son ejemplos importantísimos de las labores femeninas que siempre han sido mal pagadas y muy olvidadas como objeto de análisis histórico.

Sin embargo, no sólo en estas actividades de bajo salario y peores condiciones de trabajo es donde se puede rescatar históricamente la presencia de las mujeres latinoamericanas; los aspectos reproductivos y de la vida cotidiana son renglones relevantes en esta era en que la investigación se ha desarrollado y sobre todo, en que los enfoques se han innovado y adquirido originalidad en el uso de fuentes y en la interpretación de los problemas históri$\cos ^{17}$

\section{TEMAS CONOCIDOS}

La historia de la mujer en América Latina es en nuestros días un campo de investigación histórica en constante crecimiento $y$, aunque los estudios son todavía limitados y escasos, ya empieza a surgir un esquema general que es necesario revisar en razón de los periodos y la temática.

\section{El periodo colonial ${ }^{18}$}

Desde el punto de vista de una división temporal, el periodo colonial es el que ocupa mayor espacio (1500-1820) y el más estudiado, tanto en términos de sus temáticas como en el de los países. Como podría esperarse, los países que tienen mayor población indigena son los que cuentan con estudios más profundos, especialmente en relación con las mezclas étnicas y con los efectos que han tenido éstas en las mujeres. El hecho de que se trate de una perspectiva temporal muy amplia, tres siglos, permite algunas conclusiones preliminares sobre esta época, puesto que las mujeres indigenas fueron de capital importancia en el proceso de mezcla durante ese periodo, ya que, muy frecuentemente, eran ellas quienes tenían vástagos de los españoles; además las mezclas entre diferentes grupos eran sumamente frecuentes. Los mestizos, producto de español e indígena, eran abundantes, y una población cada vez más heterogénea creció rápidamente, a pesar de los esfuerzos de la corona, sobre todo al inicio, por detener mediante una política de prohibición los matrimonios mixtos y las mezclas étnicas. El fenómeno del mestizaje cambió la composición poblacional del continente, especialmente en países como México, Colombia, Perú y Venezuela, que son hasta la fecha los paises con mayor número de mestizos del área. La relación entre sexo y etnia es compleja y es necesario contar con más estudios sobre el caso para entenderla cabalmente, puesto que hay varias interpretaciones sobre el papel de las mujeres en su carácter de concubinas, esposas, amantes, etc., y a pesar de que el tema no ha sido ago-

\footnotetext{
${ }^{7}$ Asunción Lavrin, "Algunas consideraciones finales sobre las tendencias y los temas en la historia de las mujeres de Latinoamérica" en Las mujeres Latinoamericanas, perspectivas históricas, Asunción Lavrin, compiladora, México, Fondo de Cultura Económica, 1985, p. 347-379.

${ }_{i \AA}$ El periodo precolombino, no considerado en este ensayo, con su variedad de culturas regionales, merece un estudio particular extenso. Para alguna bibliografia básica véase: Mari Knaster, op. cit. , 1977, p. 445.
} 
tado, se puede decir que la posición de la mujer dependía en buena medida de su posición en la comunidad, más que en razón de sus ligas con un hombre, fuese éste español, indio o mulato.

Sin embargo, estos esquemas fueron cambiando durante la época colonial y pese a los esfuerzos de la corona española por conservar una sociedad estática, claramente dividida en clases, lo que existe actualmente en América Latina, es una sociedad multiétnica, dividida en grupos sociales sumamente diversos, en la que las mujeres de diferentes antecedentes socioculturales coexisten de muchos modos contradictorios, por lo que surgen las preguntas ¿qué significaba ser indígena, negra, mulata o blanca en la Hispanoamérica colonial? y ¿cómo se ha modificado esto en la actualidad? Los trabajos de investigación recientes han apuntado hacia el hecho de que aunque las mujeres de todas las clases estaban sujetas a esquemas de conducta determinados, "si bien todas las mujeres se desviaron de esta meta, el impacto de la sanción en los casos individuales de mujeres estaba condicionado por su posición de clase". 19

\section{Las mujeres de la elite}

El estatus social resultaba determinante para hombres y mujeres de la Hispanoamérica colonial y la ley establecia las diferencias de clase de manera sumamente clara, de modo que las mujeres blancas tenían frecuentemente una posición privilegiada, acceso a la riqueza, al prestigio social, al tiempo libre y a la cultura, e inclusive un cierto margen de libertad individual; en algunos casos, hasta fueron precursoras de nuevas conductas al superar las normas sociales rigidamente definidas para su clase. Al estudiar a las familias de la elite en la época colonial, es frecuente encontrar que las mujeres tenían amplia participación en el control de la riqueza y la propiedad, y aun cierto poder de decisión en los negocios familiares, en contra de las normas prescriptivas que relegaban a la mujer a la casa y a la iglesia exclusivamente. La actividad económica femenina se incrementa ante la ausencia de los hombres de la familia; algunas instituciones femeninas, como los monasterios y conventos, fueron decisivas por su enorme influencia económica en esa época. ${ }^{20}$

También debido a trabajos recientes, nos es posible ahora asomarnos a la vida sexual de las mujeres de esos tiempos. ${ }^{21}$ Aquí, lo

1" Elinor Burkett, "In dubioussisterhood: class and sex in Spanish Colonial South America" en Women and class struggle, volumen especial de Latin American Perspectives, núm. 4 núms. 1, 2, invierno y primavera, 1977, p. 445.

?1) Edith Coutourier, "Las mujeres de una familia noble: los condes de Regla de México, 1750-1830" en Asunción Lavrin, op. cit., 1985, p. 153-176. Doris Ladd, Mexican nobility at independence 1780-1826. Austin University of Texas at Austin, 1976, p. 54.

"Asunción Lavrin, "Aproximación histórica al tema de la sexualidad en el México colonial" en Encuentro, revista del colegio de Jalisco, vol. 2, núm. 1, octubre, diciembre. 1984. p. 23-40. Véase también: Varios: Familia y sexualidad en Nueva Espana. México, Secretaria de Educación Pública, Fondo de Cultura Económica, 1982. Este volumen se ocupa de aspectos específicos de la compleja relación entre sexo y familia. Otro estudio importante es el de Silvia Arrom; basándose en casos del archivo judicial. Arrom ha rastreado las actitudes de la mujer respecto al divorcio en La mujer ante el divorcio eclesiástico, México, SEP, 1976, Sepsetentas, núm. 251. 
importante es hacer la diferencia entre las prácticas prescritas por la legislación y las prácticas reales. Tanto las leyes civiles como eclesiásticas fueron muy cuidadosas en establecer lo permitido y lo no permitido para determinar qué era y qué no era pecado. Para los historiadores modernos, fuentes como los juicios inquisitoriales, sermones, manuales de confesión, cartas personales, panfletos sobre conductas maritales y archivos judiciales donde se llevaba relación de los juicios maritales, son un tesoro de información que permite perfilar una imagen, más exacta, de las conductas sexuales femeninas. Otros aspectos, como la violación, la homosexualidad y otras prácticas, también se han analizado, en especial la compleja relación entre clases y delincuencia sexual. ${ }^{22}$

\section{El estatus legal}

El hecho de que América Latina fuera colonia durante un periodo tan largo, con un sistema burocrático altamente centralista, ha dejado a los historiadores con montañas de material que puede ser aprovechado para el análisis de la situación de las mujeres en la colonia. A pesar de que este material hace énfasis en el punto de vista del estado, se han hecho varios análisis sobre las prácticas concretas en contraste con la legislación. ${ }^{23}$

Fenómenos como las dificultades matrimoniales, el divorcio, la separación, el adulterio, la delincuencia femenina y la prostitución, así como las disputas sobre propiedades, herencias, etc., etc., son algunos de los temas importantes que podrian estudiarse. Las recopilaciones o colecciones de leyes que la Corona ordenó periódicamente no son sino una fuente importante de este tipo de trabajo así como los ordenamientos legales dados por funcionarios de menor jerarquía. Las colecciones de leyes son asimismo una buena fuente para revisar los ordenamientos legales practicados en el periodo, especialmente en relación a dotes y a las instituciones que la corona española estableció para proteger a las mujeres sin dote. $^{24}$

En general, los estudios sobre las mujeres de la época colonial se han centrado más sobre las mujeres de la elite, sobre todo porque las fuentes son más abundantes para este grupo, pero habria que renovar técnicas y buscar nuevas fuentes para el estudio de otros grupos: indígenas, negras, mulatas y miembros de las castas.

\section{Las mujeres en la sociedad}

Así pues, la imagen de los plácidos y somnolientos tiempos coloniales está desapareciendo en la medida en que se investiga más a

\footnotetext{
$\because$ Varios artículos con esta temática aparecieron en La memoria y el olvido, Segundo simposio de historia de las mentalidades. México, Instituto Nacional de Antropologia e Historia, 1985. Para el caso brasileño véase A. J. Rusell Wood, "Dowries helped reduced domestic instability, ilegitimacy and prostitution in Bahia" en Lewis Hanke editor, Latin America: a historical reader, Boston, Little Brown and Co., 1974, p. $181-187$.

23 Un buen estudio amplio y completo es el del profesor Charles Boxer, Women in overseas Iberian Expansion, Nueva York, Oxford University Press, 1977.

24 Josefina Muriel, Los recogimientos de mujeres, México, UNAM, 1974; Asunción Lavrin y Edith Coutourier, "Dowries and Wills: a view of women's socioeconomic role in Guadalajara and Puebla, 1640-1790", Hispanic American Historical Review, núm. 59, mayo 1979 , p. 280-304.
} 
fondo en la sociedad colonial, que aumenta en complejidad y variedad temática. Sin embargo, el papel de la mujer en la multiétnica sociedad colonial no ha sido suficientemente estudiado y el paso lógico siguiente, la reconstrucción de los esquemas familiares, para evaluar el complicado proceso de la marginalidad o movilidad en relación con las mujeres y su grupo étnico, está aún en una etapa incipiente. El estudio de las mujeres que no pertenecen a la elite es, prácticamente, un área desconocida y necesitamos profundizar en la relación de la mujer y el intrincado proceso de mestizaje, porque las variantes de tiempo y región pueden proporcionarnos una imagen especialmente compleja de estas centurias, de relaciones multiétnicas, en las que las mujeres fueron determinantes. ${ }^{25}$

\section{El periodo nacional}

El siglo XIX o el periodo nacional de la historia latinoamericana (1820-1920) no está bien provisto de temas para investigar, a pesar de que las fuentes dé investigación son abundantes $y$, en ocasiones, de acceso más fácil que las del periodo anterior. En el siglo XIX, en sus inicios, el sindrome de la "mujer heroína" es sumamente notable y hay numerosos biógrafos de las mujeres independentistas. Las guerras de liberación en Hispanoamérica arrojaron cierta luz sobre sus vidas, sobre todo de aquéllas que estuvieron en contacto con héroes y caudillos locales, con el propósito primordial de glorificar la actitud proindependentista de las mujeres. Un enfoque quizá más productivo sería el de tratar de encontrar cuáles fueron los cambios que la independencia trajo a su vida. Por una parte, sabemos que especialmente entre las mujeres de las clases altas urbanas, los patrones de consumo se incrementaron y, sin embargo, no sabemos cuáles fueron los efectos específicos que para las mujeres tuvieron las crisis económicas que las repúblicas latinoamericanas tuvieron que sortear, y en qué medida estas crisis significaron un detrimento en las expectativas que las mujeres tenian de mejorar su situación con la independencia.

Aunque parezca sorprendente, en general, las mujeres tuvieron mejores oportunidades de mejorar su suerte hacia el fin del periodo colonial que a principios del siglo XIX, cuando los problemas económicos de las nuevas repúblicas frustraron frecuentemente las esperanzas de los grupos dirigentes del periodo anterior ${ }^{26}$

El siglo XIX nos proporciona aun otro tema de investigación en relación con la situación legal de la mujer. Precisamente con el cambio de una legislación colonial a una legislación liberal, podemos evaluar cuál era el papel que los nuevos estados nacionales adscribieron a las mujeres y cuán poco igualitarios eran los dere-

\footnotetext{
25 Para una discusión sobre las tendencias en la historia social mexicana, véase: Marcello Carmagnani, "The inertia of Clio: the social history of Colonial Mexico" en Latin American Research Review, vol. XX, núm. 1, 1985, p. 149-166. Para el caso latinoamericano en su conjunto véase: Fred Bonner, "Urban Society in colonial Spanish America: research trends" en Latin American Research Review, vol. XXI, núm. 1, 1986, p. 7-72.

${ }_{26}$ Silvia Arrom, The women of Mexico City 1790-1857, Stanford, California, Stanford University Press, 1985, p. 216.
} 
chos individuales de las mujeres en comparación con los de los hombres. El análisis de los códigos legales en los cuales las relaciones familiares se reglamentaban, ha probado ser un rico campo para evaluar el estatus legal de la mujer mexicana de ese periodo; podrían llevarse a cabo estudios similares en otros paises.

Por otra parte, la batalla política que tiene lugar para organizar los estados nacionales en un proceso de larga duración en el siglo $x I X$ latinoamericano $y$, en algunos casos, la confrontación entre el estado y la iglesia era inevitable, como en Colombia y México, cuya inestabilidad política durante ese periodo puede proporcionar excelentes datos para medir la relación entre el desorden social y la cohesión de las redes familiares, y puntualizar el papel de las mujeres en estas últimas. Esto constituye un aspecto importante, puesto que ya ha sido documentado que la familia, en el siglo XIX, tuvo un papel preponderante en América Latina durante el proceso de consolidación capitalista, pues era a través de las redes de familia como se arreglaban la mayoría de los negocios y se acrecentaban las propiedades territoriales. ${ }^{28}$

Sin embargo, el problema central para la historia de las mujeres latinoamericanas del siglo XIX es su papel en la formación de los estereotipos y conductas sociales que sustituyeron los hábitos y la moral colonial. Una vez desaparecido el rígido sistema de leyes que regulaba los aparejamientos interétnicos, ¿en qué medida cambiaron las actitudes femeninas hacia el matrimonio y la reproducción? El estudio precursor sobre el matrimonio en la Cuba decimonónica y sus relaciones con los problemas de clase, especialmente de negros libres, está basado en el estudio de conductas "aberrantes" y se hizo usando los archivos judiciales. ${ }^{29}$ Este estudio ha servido para mostrar la mecánica de las relaciones entre clase y color en una floreciente economia de plantación. Estudios similares y específicos de otras regiones del área se hacen necesarios, pues aunque Cuba permaneció como colonia hasta muy entrado el siglo XIX, se podrían hacer algunas comparaciones con las nuevas repúblicas independientes.

Para el fin del siglo XIX, los temas de investigación son muy abundantes; el inicio de la integración de las mujeres al trabajo fabril nos proporciona la oportunidad de analizar las formas en que se dividia el trabajo por sexos en el proceso productivo de cada industria, y nos enseña cuáles eran los oficios que se consideraban propios de las mujeres. Hay algunos casos obvios, como el de las profesoras de primaria, oficio que aparece en manos femeninas desde el siglo XVI, cuando la enseñanza básica para las niñas se llevaba a cabo en hogares privados, y mujeres de cierta edad enseñaban a las jóvenes bordado, canto, música, cómo leer y otras labores; sin embargo, otros campos de la actividad femenina han tenido menos continuidad que la enseñanza y es preciso señalar cuáles son los cambios que, en este aspecto, se van dando a través del tiempo, particularmente a fines del siglo pasado.

27 Ibid.

-8 Enrique Florescano, coordinador, Origenes y desarrollo de la burguesia en America Latina 1700-1955. México. Editorial Nueva Imagen, 1985.

2) Verena Martinez Alier, Marriage, class and colour in nineteenth century Cuba. A study of racial attitudes and sexual values in a slave society, Cambridge University Press. 1974. 
El creciente número de mujeres que se integra a un tipo de trabajo asalariado en las fábricas de textiles y de tabaco, en imprentas, como enfermeras, secretarias, etc., puede proporcionarnos material suficiente para establecer una imagen general de la vida de las mujeres en las zonas urbanas en ese periodo y sobre su participación en las formas iniciales de organización laboral: cooperativas, uniones, clubes, sociedades mutualistas y demás. ${ }^{30}$ En tanto que el de textiles es un sector bien conocido por su beligerancia, la participación de mujeres en él, con demandas claramente orientadas hacia su propio beneficio, está aún poco documentada. Habria que saber hasta qué grado las mujeres que compartian un trabajo común en la fábrica fueron determinantes en la formación de una conciencia de clase y en qué medida sus peticiones incluían demandas específicas para el mejoramiento de la vida de la mujer trabajadora. Si bien los periódicos incluían frecuentemente artículos sobre mujeres, aún no se ha formado una imagen 'general del moyimiento latinoamericano de trabajadoras y sus ligas internacionales, como un ejemplo del proceso de construcción de clase y de género, en el que se señalen las diferencias regionales específicas.

\section{El siglo $x x$}

El hecho de que el siglo $\mathrm{xx}$ haya sido significativo en muchos países latinoamericanos por el incremento de la participación femenina en la fuerza de trabajo en las áreas urbanas y por la emigración masiva hacia esas mismas áreas, nos indica que es necesario estudiàr las etapas, variedades y ciclos del crecimiento de la fuerza femenina de trabajo. También deben entenderse los cambios en los esquemas del trabajo femenino y correlacionar las dimensiones de la fuerza de trabajo con el tipo de trabajo y los ciclos productivos de las economías regionales. Asimismo es necesario conocer las variantes en las formas de integración de la mujer a la fuerza de trabajo, a través del tiempo, especialmente en aquellos paises en que las mujeres iniciaron su integración -aunque en pequeño número-, hace un siglo: Argentina, algunas regiones de Brasil, Uruguay, Chile, Colombia o México.

Otro tema importante de análisis en el siglo xx de América Latina es el que se refiere a la participación de la mujer en los movimientos sociales. Desde las tempranas huelgas de obreros textiles en Colombia, México o Brasil, hasta la movilización masiva actual en América Central, su papel ha sido sumamente activo en las demandas de sus derechos políticos y sociales, como ciudadanas y como mujeres.

El hecho de que en casos como el de la Revolución mexicana, el de la Violencia colombiana o la Revolución cubana o nicaragüense, 'las mujeres hayan tenido una participación política, que no sigue necesariamente las líneas de las estructuras partidarias dominadas por los varones, no debe impēdir el análisis de las for-

${ }^{30}$ CEHSMO, La mujer y el movimiento obrero mexicano en el siglo XIX, Antologia de la prensa obrera, México, Centro de Estudios Históricos del Movimiento Obrero Mexicano, 1975. 
mas de participación política femenina durante el presente siglo, puesto que este fenómeno bien puede ser uno de los más importantes de la América Latina contemporánea.

\section{TeMAS DE ESTUdio}

Algunos de los prōblemas apuntados anteriormente, e incluso algunos otros, no encontrarán contestación a menos que superemos una concepción positivista y ampliamente difundida, según la cual la historia sólo consiste en una aburrida lista de nombres y fechas. Unicamente si se logra integrar un enfoque interdisciplinario podemos plantear preguntas contemporáneas a los datos históricos y obtener respuestas significativas.

Dado que el estudio de la historia de la mujer es un campo relativamente nuevo en América Latina, éste es el momento oportuno para indicar algunas pautas que las investigaciones del tema podrían seguir para obtener una imagen real de la historiografía de la mujer.

\section{La demografia histórica}

Puesto que históricamente hablando las mujeres han estado sobre todo dedicadas a la reproducción, es importante conocer sus ciclos de vida en relación con el matrimonio y/o reproducción, y establecer las variantes regionales y cronológicas de dichos ciclos entre mujeres de diferentes clases y regiones.

Al conocer las variantes en los ciclos reproductivos, también estaremos en la posibilidad de conocer mejor las variaciones históricas de la familia y avanzar de la "historia familiar" a la historia de la familia como institución. Es pues necesario, analizar y comparar las variaciones en los esquemas de formaciones familiares a través del tiempo, en especial en términos de tamaño, raza y región. Digamos, por ejemplo ¿cuántos miembros tenían las familias de clase alta en Bahía, en oposición a los miembros de las familias esclavas? ¿Cómo se organizaba la unidad doméstica? ¿Cómo se comparan estos problemas con grupos similares en Lima o Buenos Aires?, etcétera.

Temas como el anterior nos proporcionarán esquemas de las formaciones familiares y si analizamos estos temas dentro de un contexto histórico determinado, podremos entender mejor las funciones sociales de la familia, los papeles asignados a cada uno de sus miembros y, en especial los de las mujeres.

Esto resulta importante porque debemos entender que la ilegitimidad es más bien un concepto de origen europeo, y que otras formas de aparejamiento como el matrimonio condicionado, las uniones paralelas o los hijos fuera del matrimonio, todos ellos fenómenos muy abundantes en América Latina, pueden responder a que las ligas familiares, las uniones matrimoniales y el concepto mismo de familia obedecen en América Latina a esquemas propios diferentes de los de la Inglaterra victoriana, que frecuentemente se han tomado como único modelo.

\section{Historia de las mentalidades}

Otro campo importante de investigación, especialmente útil 
para la historia de las mujeres, es el de la historia de las mentalidades, puesto que se ocupa de un proceso de largo alcance como el de la construcción de valores y la construcción de papeles asignados a hombres y mujeres. Si estamos interesados en descubrir cuáles han sido los cambios en las conductas y hábitos sexuales y en cómo se construyen socialmente las categorias de género: "hombre", "mujer", es a través de un análisis de las tendencias de larga duración en las conductas masculinas y femeninas como podremos evaluar qué es lo que se consideró propio de hombres y propio de mujeres en un periodo determinado y, sobre todo, cómo fue cambiando lentamente en el tiempo, y cómo estos cambios y estas permanencias actuaron en la vida de las mujeres.

Los valores se transmiten por un proceso educacional que no se limita a la instrucción escolar. La niña aprende a "ser femenina" mucho antes de pisar siquiera una escuela.

Por lo tanto, no debemos poner atención únicamente a los valores que se transmiten en la escuela, sino que es necesario hacer hincapié especialmente en la forma en que se transmiten los valores por medio de la familia, el estado o la iglesia, y en tiempos recientes por los medios de comunicación. La ventaja de analizar históricamente este proceso es la de poder evaluar la permanencia y la validez temporal de los códigos seculares, así como los elementos que favorecen el cambio. Son fuentes particularmente valiosas para el análisis los manuales para la crianza de los niños, los sermones, los programas escolares, las revistas para mujeres y familias, asi como las novelas, las canciones o los libros de historietas.

\section{La psicología social en la historia}

Los valores no se refuerzan solamente a través de las instituciones; son los individuos los que los refuerzan con cierto tipo de comportamiento. En las mujeres, este proceso es particularmente importante porque los valores que rigen sus vidas no han sido establecidos por mujeres, aunque son las mujeres, especialmente en su papel de madres y de educadoras, las que refuerzan, transmiten y reproducen estos valores de generación en generación. Pocos trabajos históricos han señalado las características de este proceso y desde luego, prácticamente no existen en el caso latinoamericano. ${ }^{31}$

Las mujeres han tenido un espacio limitado para expresarse y si queremos saber cómo perciben las mujeres sus funciones y cómo éstas dan forma a sus conductas personales, necesitamos utilizar algunas de las pocas fuentes en las cuales existe cabida para la voz de las mujeres: la literatura, la tradición oral y otras formas de cultura popular, incluso las historietas. Estas fuentes serían muy útiles para estudiar las variantes históricas en el concepto social de las mujeres, puesto que cubren aspectos de conducta que no aparecen en otras fuentes.

\section{Creando una conciencia histórica}

La historia no es, o más bien no debería ser, el tipo de conoci-

3 Marie Françoise Levy, De Mères en filles. L'èducation des françaises 1850 1980. París, Calman Levy, 1984. 
miento restringido, accesible sólo para un número muy reducido de personas "iniciadas". Esta clase de concepción de la historia implica una visión estática del pasado, útil para conmemorar héroes y batallas en las ceremonias cívicas, visión que desafortunadamente ha prevalecido en América Latina, especialmente en los círculos de gobierno y política. Esta imagen de la historia, solemne y aburrida provoca el desinterés de la mayoría de la gente.

Un primer paso inicial para superar este enfoque paternalista hacia la historia y el conocimiento histórico en América Latina es reforzar la idea de que la historia es algo que está en continuo cambio, que está presente en la vida diaria y que no se trata simplemente de una lista de nombres fácilmente olvidables. Es necesario reflexionar sobre el hecho de que la historia está presente en nuestra vida cotidiana: casa, comida, ropas, hábitos sociales, formas de trabajo, etc., son producto de una experiencia histórica particular; y debemos reforzar la idea de que aprender historia significa aprender la relación entre cambio y continuidad y entender quẻ algunas cosas cambian rápidamente y otras a ritmo lento. Explicar el porqué de permanencias y cambios es la verdadera tarea histórica.

Es preciso destacar también que los individuos, los grupos sociales y los países, tienen un papel en la historia. Los acontecimientos no "le ocurren" a la gente, sino que son los grupos, los individuos, hombres, mujeres, los que son la historia. Por lo tanto, también son los individuos los que determinan lo que es o no es relevante en la historia. Porque en momentos diferentes son diferentes los temas y problemas que se consideran históricamente relevantes, nos interesamos ahora en la historia de las mujeres.

En el caso latinoamericano, la mayoría de la gente no ve de esta manera la información con respecto a la historia. Existe, en cambio, la idea común de que la historia es algo oficial, despegado de las personas, e irrelevante para la vida cotidiana. En lo que respecta a las mujeres, esto está aún más acentuado porque sus vidas, sus intereses y sus actividades han sido consideradas tradicionalmente como irrelevantes.

Al crear una conciencia histórica es neceario darse cuenta que la historia no es un pasado muerto, sino más bien una construcción viva y presente del conocimiento en relación con temas y problemas que tienen importancia en la vida personal y colectiva actual.

\section{Dónde encontrar materiales $e$ información}

En oposición a otros científicos sociales que tienen que generar sus propios datos, los historiadores tienen que generar una interpretación y organización de los mismos, pero en general, cuentan con ricos materiales, especialmente en el nivel regional, donde los archivos parroquiales, municipales, cartas personales y otros papeles "poco importantes" son, de hecho, fuentes históricas, así como la memoria de ancianos.

El valor de estas fuentes en la investigación histórica ha sido reconocido recientemente. Los historiadores locales son casi siempre fuentes insustituibles para reconstruir la vida cotidiana de sus pequeñas comunidades. Así pues, existe una necesidad creciente de reinterpretación en América Latina donde estas fuentes existen, y sin embargo, se han usado mínimamente. 


\section{De receptivo a activo en la investigación histórica}

Además de revaluar las fuentes potenciales, también es muy importante crear la conciencia de que todo el mundo es un historiador potencial en la medida en que sea capaz de interpretar su pasado, de recordarlo y encontrar su significado en razón del presente. En cuanto a las mujeres esto tiene particular importancia porque han sido las mujeres quienes han estado tradicionalmente ligadas a la casa, los niños, el matrimonio, los nacimientos y otros acontecimientos que forman parte de la memoria familiar. Este lazo con los acontecimientos memorables de la vida familiar, puede ser particularmente útil para favorecer su interés y participación en conservar y usar como documentos históricos cartas, fotografías, objetos personales y más que nada sus recuerdos, su memoria colectiva y sus tradiciones orales. La evaluación, discusión y comparación de estas experiencias femeninas, las llevaría inevitablemente a una imagen más auténtica de sí mismas $\mathrm{y}$, por lo tanto, a uná autopercepción menos disminuida.

\section{Importancia de la voz de las mujeres}

Es precisamente la autopercepción de las mujeres, expresada por sus propias voces, la que es necesario escuchar para reformular y modificar la tradicional imagen de la mujer como ser dependiente, carente de iniciativa y de capacidad. En América Latina, las mujeres tienen presencia y voz en los asuntos familiares, pero su voz no se considera importante más allá del ámbito doméstico, y por lo tanto se tiene que superar la idea de que la vida privada es poco importante, y que lo que importa únicamente es la vida pública, es decir, la vida masculina. Debemos además, establecer las relaciones entre ambas y mostrar la falsedad de su separación.

En América Latina, donde el analfabetismo es aún un fenómeno muy extendido, no es en los libros donde las mujeres aprenderán y percibirán su autoimagen. La imagen de lo que es femenino se exhibe en revistas, fotografías, anuncios, películas, radio, historietas y televisión. En la mayoría de los casos las mujeres aparecen como indefensas, dependientes y como criaturas más bien estúpidas que no pueden ni siquiera elegir y mucho menos poner en práctica sus propias decisiones. Debemos superar la imagen tradicional, de telenovela, para restaurar y dignificar la imagen colectiva y para dar a las nuevas generaciones la posibilidad de elección y de conciliación entre las diferentes opciones de familia, carrera y otras. Lo más importante es caer en la cuenta de que son las mujeres quienes, si tienen oportunidad, tendrán mucho que decir sobre sí mismas; hombres y mujeres, jóvenes y viejos podrian aprender mucho si los escucharan con atención. ${ }^{32}$

\section{HISTORIA, UNA DISCIPLINA DE INTERPRETACIÓN}

El estar informado de la importancia de la historia de las mujeres es un fenómeno reciente, restringido en el caso de América

"Deirdre Beddoe, Discovering Women's history, a practical manual, Londres, Pandora Press, 1983. 
Latina, a las instituciones de investigación y a los escasos grupos de mujeres organizadas que se interesan por su pasado.

No es necesariamente en los círculos académicos donde crece, lentamente, esta nueva actitud latinoamericana hacia la historia, sino en los grupos autónomos de mujeres que tienen la iniciativa y el entusiasmo para explorar su pasado; sin embargo, falta mucho por hacer. Debemos superar e ir más allá del "club universitario" y crear una liga estable y amplia entre investigadores y audiencias amplias. Además, conferencias, materiales gráficos y audiovisuales, grupos de discusión, visitas guiadas, entrevistas, son otras formas alternativas de conocer la historia femenina.

Otra área en la que debe incrementarse la atención prestada a la historia de la mujer es la de los programas escolares. Es necesario establecer cursos sobre mujeres en la historia, en las escuelas medias y preparatorias, y establecer también cursos y equipos universitarios de investigación que además rescaten textos con los que se conformen libros de curso, inexistentes en la actualidad.

Sin embargo, no es sólo en la educación formal en la que podremos aprender historia de las mujeres. Las universidades latinoamericanas no son muy numerosas y es generalmente a través de la educación informal como la mayoría de la gente aprende las funciones femeninas.

Los valores familiares se transmiten desde la primera infancia; al niño y a la niña se les señalan sus diferencias y se les impone una conducta determinada mucho antes de asistir a la escuela.

La familia es probablemente la primera fuente de educación informal; sin embargo, los valores familiares son producto de los valores sociales que se transmiten por radio, televisión, cine, etc., y por ese vehículo que parece ser un fenómeno tipicamente latinoamericano: la fotonovela, cuyos lectores son mayoritariamente las mujeres. Para muchas mujeres latinoamericanas este es el unico material de lectura al que tienen acceso. Por estos mismos medios el trabajo histórico puede diseminarse y así alcanzar una percepción diferente del papel de la mujer en la historia; la tarea es impostergable y desde luego urgente. 